\title{
Vorhofflimmern
}

\section{Ältere Frauen haben ein höheres Schlaganfallrisiko als Männer}

Avgil Tsadok $M$, Jackevicius $C A$ Rahme E et al. Sex differences in stroke risk among older patients with recently diagnosed atrial fibrillation. JAMA 2012; 307: $1952-8$

Abbildung 1 Schlaganfallrisiko bei Vorhofflimmern. Ältere Frauen haben ein höheres Risiko.
Fragestellung: Gibt es bei älteren Menschen mit Vorhofflimmern Geschlechtsunterschiede bezüglich des Schlaganfallrisikos?

Hintergrund: Etwa 1\% der Bevölkerung leidet unter Vorhofflimmern. Die Häufigkeit des Vorhofflimmerns nimmt mit dem Alter dramatisch zu. Patienten mit Vorhofflimmern haben ein fünffach erhöhtes Schlaganfallrisiko. Bisher durchgeführte epidemiologische Studien zeigten entweder ein höheres Risiko für Frauen oder ein identisches Geschlechtsverhältnis, daher war es notwendig, weitere epidemiologische Daten für diese Fragestellung zu gewinnen.

Patienten und Methodik: Es handelt sich um eine populationsbezogene Kohortenstudie von Patienten im Alter von 65 Jahren und älter, die zwischen 1998 und 2007 wegen Vorhofflimmern ins Krankenhaus eingewiesen wurden. Die Erhebung stützt sich auf die Datenbanken der Krankenhäuser, der nachbehandelnden Ärzte und der in der Folgezeit ausgestellten Rezepte. Der primäre Endpunkt war das Schlaganfallrisiko.

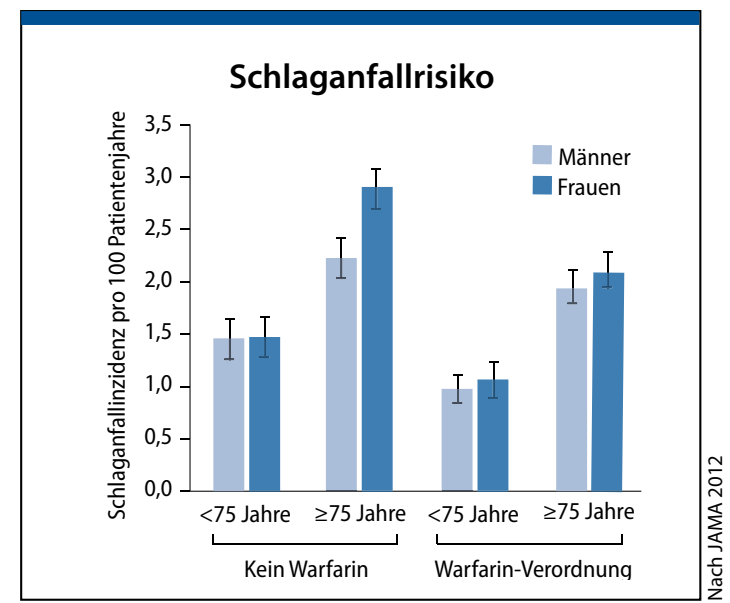

Ergebnisse: Die Kohorte bestand aus 39.398 Männern und 44.115 Frauen. Zum Zeitpunkt der Aufnahme in das Krankenhaus waren Frauen älter und hatten einen höheren CHADS2-Score (1,99 vs. 1,13). 30 Tage nach der Entlassung bekamen 58,2\% der Männer und 60,6\% der Frauen eine orale Antikoagulation mit Warfarin. Die Adhärenz der Behandlung mit Warfarin war in beiden Behandlungsgruppen gut. Das Risiko eines Schlaganfalls betrug bei Frauen 2,02 pro 100 Patientenjahre, bei Männern 1,61 pro 100 Patientenjahre (Abbildung 1). Dieser Unterschied war statistisch signifikant. In einer multivariablen Regressionsanalyse hatten Frauen ein um 14\% höheres Schlaganfallrisiko als Männer, auch wenn für andere Risikofaktoren und vaskuläre Erkrankungen korrigiert wurde.

Schlussfolgerung: Ältere Frauen mit Vorhofflimmern haben ein höheres Schlaganfallrisiko als Männer.

Kommentar: Diese epidemiologische Studie belegt, was frühere Studien ebenfalls gezeigt hatten, dass nämlich ältere Frauen ein höheres Schlaganfallrisiko bei Vorhofflimmern haben als Männer. Daher ist es gerechtfertigt, weibliches Geschlecht in den RisikoScore CHA2DS2-VASc2 aufzunehmen. Dies mag zum Teil daran liegen, dass Frauen im Alter über 75 Jahren signifikant seltener mit Vitamin-K-Antagonisten behandelt werden als Männer. Wenn sie mit Warfarin behandelt werden, ist die Adhärenz an die Behandlung deutlich besser als bei Männern. Mögliche Erklärungen für die Unterschiede könnten daran liegen, dass Frauen häufiger allein leben und dies dann gegebenenfalls die regelmäßige Kontrolle der INR-Werte erschwert.

Hans-Christoph Diener, Essen

\section{InFo Neurologie und Psychiatrie wertet laufend unter anderem folgende Zeitschriften aus:}

Acta Psychiat Scand • Am J Psychiatry • Ann Neurol • Arch Gen Psych • Arch Neurol • Arzneimitteltherapie • Behav Res Ther - Biol. Psychiatry • BMJ • Brain • Brit J Psychiatry • Cephalalgia • Cerebrovasc Dis • Compr Psychiat • Epilepsia • Eur Arch Psy Clin N • Europ Neurology • Eur Psychiatry • J Clin Psychiatry • J Nerv Ment Dis $\cdot J$ Neurol • J Neurol Neurosurg Psychiatry -J Personal Dis • J Psychiat Res • JAMA • Headache • Lancet • Lancet Neurol • Mol Psychiatry • N Engl J Med • Nat Med • Nature • Nervenarzt • Neurology • Neuropsychopharmacol • Pain • Pharmacopsychiatry •PPmP•Proc Natl Acad Sci USA • Psychiat Res • Psychol Med • Psychopharmacology $\cdot$ Science $\cdot$ Stroke $\cdot$ Zeitschrift für PPP 